\title{
SOME EXPLICIT SOLUTIONS FOR THE JOINT DENSITY OF THE TIME OF RUIN AND THE DEFICIT AT RUIN
}

\author{
BY
}

\author{
DAVID C.M. Dickson
}

\begin{abstract}
Using probabilistic arguments we obtain an integral expression for the joint density of the time of ruin and the deficit at ruin. For the classical risk model, we obtain the bivariate Laplace transform of this joint density and invert it in the cases of individual claims distributed as Erlang(2) and as a mixture of two exponential distributions. As a consequence, we obtain explicit solutions for the density of the time of ruin.
\end{abstract}

\section{INTRODUCTION}

In recent years a number of new explicit solutions for finite time ruin problems have appeared in the literature. See, for example, Drekic and Willmot (2003), Dickson and Willmot (2005), Dickson et al (2005) and Garcia (2005). In this paper we extend results given in some of these papers by considering the joint distribution of the time of ruin and the deficit at ruin in the classical risk model. In particular, we derive explicit solutions for the joint density of these quantities for two individual claim amount distributions - Erlang(2) and a mixture of two exponential distributions.

It is well known that in the classical risk model with exponentially distributed individual claims, the distribution of the deficit at ruin is independent of the time of ruin. See, for example, Gerber (1979). Otherwise results in the literature on the joint distribution of the time of ruin and the deficit at ruin in the classical risk model are restricted to Laplace transforms. By writing the 'penalty' function in the well-known Gerber-Shiu function (Gerber and Shiu (1998)) as $w(x, y)=e^{-s y}$, the Gerber-Shiu function becomes the bivariate Laplace transform of the time of ruin and the deficit at ruin. Dickson and Drekic (2006) and Cheung et al (2007) exploit this fact to obtain the form of the bivariate density of the time of ruin and deficit at ruin for certain individual claim amount distributions. However, their solutions are expressed in terms of functions that are not identified, but whose Laplace transforms are known but difficult to invert. In this paper we do not attempt to invert these functions, but we do exploit the structural forms of bivariate densities obtained in these papers. In the special case when the initial surplus is 0 , an expression (equation (2) 
below) for the bivariate Laplace transform of the time of ruin and the deficit at ruin exists, and this can be inverted using the techniques of Dickson and Willmot (2005) to give an expression for the joint density in terms of the density function of aggregate claims.

We start with some general results. Let $\{U(t)\}_{t \geq 0}$ denote a surplus process and let $T$ denote the time of ruin. Define

$$
W(u, y, t)=\operatorname{Pr}(T \leq t,|U(T)| \leq y \mid U(0)=u),
$$

to be the defective joint distribution function of the time of ruin and the deficit at ruin, and let

$$
w(u, y, t)=\frac{\partial^{2}}{\partial y \partial t} W(u, y, t)
$$

be the defective joint density.

By conditioning on the time and the amount of the first drop of the surplus process below its initial level we have

$$
\begin{aligned}
W(u, y, t)= & \int_{\tau=0}^{t} \int_{x=0}^{u} w(0, x, \tau) W(u-x, y, t-\tau) d x d \tau \\
& +\int_{\tau=0}^{t} \int_{x=u}^{u+y} w(0, x, \tau) d x d \tau .
\end{aligned}
$$

Taking the partial derivative of $W(u, y, t)$ with respect to $t$ we obtain

$$
\begin{aligned}
\frac{\partial}{\partial t} W(u, y, t)= & \int_{\tau=0}^{t} \int_{x=0}^{u} w(0, x, \tau) \frac{\partial}{\partial t} W(u-x, y, t-\tau) d x d \tau \\
& +\int_{x=u}^{u+y} w(0, x, t) d x,
\end{aligned}
$$

and then taking the partial derivative with respect to $y$ we obtain

$$
w(u, y, t)=\int_{\tau=0}^{t} \int_{x=0}^{u} w(0, x, \tau) w(u-x, y, t-\tau) d x d \tau+w(0, u+y, t) .
$$

Further, we define

$$
w(u, t)=\int_{0}^{\infty} w(u, y, t) d y
$$

to be the defective density of $T$, and let $\psi(u, t)=\operatorname{Pr}(T \leq t)$. Next, let

$$
\bar{\psi}(u, t)=1-\psi(u, t)=1-\int_{0}^{t} w(u, s) d s
$$


denote the survival probability to time $t$ from initial surplus $u$. Then

$$
\bar{\psi}(u, t)=\int_{\tau=0}^{t} \int_{y=0}^{u} w(0, y, \tau) \bar{\psi}(u-y, t-\tau) d y d \tau+\bar{\psi}(0, t),
$$

and differentiation with respect to $t$ gives

$$
\begin{aligned}
-w(u, t)= & \int_{0}^{u} w(0, y, t) d y \\
& -\int_{0}^{t} \int_{0}^{u} w(0, y, \tau) w(u-y, t-\tau) d y d \tau-w(0, t),
\end{aligned}
$$

or, equivalently,

$$
w(u, t)=\int_{0}^{t} \int_{0}^{u} w(0, y, \tau) w(u-y, t-\tau) d y d \tau+\int_{u}^{\infty} w(0, y, t) d y .
$$

All the above formulae apply equally to a Sparre Andersen risk model and to the classical risk model. Our aim in this paper is to apply these formulae to the classical risk model to obtain explicit solutions for quantities such as $w(u, y, t)$ and $\psi(u, t)$. Our approach will be to use Laplace transforms.

\section{BACKGROUND}

In the classical risk model, claims occur as a Poisson process, and we let $\lambda$ denote the parameter of this process. Let $F$ denote the distribution function of individual claim amounts and let $f$ denote the density function. Let $g(\bullet, t)$ denote the density of aggregate claims over $(0, t)$ so that

$$
g(x, t)=\sum_{n=1}^{\infty} e^{-\lambda t} \frac{(\lambda t)^{n}}{n !} f^{n^{*}}(x)
$$

where $f^{n^{*}}$ denotes the $n$-fold convolution of $f$ with itself. Let $c$ denote the premium income per unit time which we assume to be received continuously, and we assume that $c$ exceeds the expected aggregate claim amount per unit time.

For the classical risk model, Lundberg's fundamental equation is

$$
\lambda+\delta-c t=\lambda \int_{0}^{\infty} e^{-t x} f(x) d x,
$$

where $\delta>0$, and Gerber and Shiu (1998) show that there is a unique positive solution to this equation, which we denote by $\rho$. Further, from formula (2.26) of Gerber and Shiu (1998) we have

$$
\int_{0}^{\infty} \int_{0}^{\infty} e^{-s y-\delta t} w(0, y, t) d y d t=\frac{\lambda}{c} \int_{0}^{\infty} e^{-\rho t} \int_{t}^{\infty} e^{-s(y-t)} f(y) d y d t .
$$


A key result in our analysis is the Laplace transform relationship given by Dickson and Willmot (2005). They show that for two functions $A$ and $B$, if

$$
\tilde{A}(\rho) \stackrel{\text { def }}{=} \int_{0}^{\infty} e^{-\rho t} A(t) d t=\tilde{B}(\delta) \stackrel{\text { def }}{=} \int_{0}^{\infty} e^{-\delta t} B(t) d t
$$

then

$$
B(t)=c e^{-\lambda t} A(c t)+\int_{0}^{c t} \frac{x}{t} g(c t-x, t) A(x) d x
$$

Cheung et al (2006) obtain general expressions for $w(u, y, t)$ for two types of individual claim amount distribution - a combination of exponential distributions and mixed Erlang distributions. Specifically, they show that if $f(x)=$ $\sum_{i=1}^{n} p_{i} \alpha_{i} \exp \left\{-\alpha_{i} x\right\}$ where $\sum_{i=1}^{n} p_{i}=1$ and each $p_{i} \neq 0$ then

$$
w(u, y, t)=\sum_{i=1}^{n} h_{i}(u, t) \alpha_{i} \exp \left\{-\alpha_{i} y\right\}
$$

where the Laplace transform of $h_{i}(u, t)$ is known for $i=1,2, \ldots, n$. Similarly, if

$$
f(x)=\sum_{i=1}^{n} q_{i} \frac{\beta^{i} x^{i-1} \exp \{-\beta x\}}{\Gamma(i)}
$$

where $\sum_{i=1}^{n} q_{i}=1$ and each $q_{i} \geq 0$ then

$$
w(u, y, t)=\sum_{i=1}^{n} \eta_{i}(u, t) \sum_{k=n+1-i}^{n} q_{k} \frac{\beta^{k-n+i} y^{k-n+i-1} \exp \{-\beta y\}}{\Gamma(k-n+i)}
$$

where the Laplace transform of $\eta_{i}(u, t)$ is known for $i=1,2, \ldots, n$. In this paper we consider special cases of these distributions - an Erlang(2) distribution and a mixture of two exponential distributions - and we obtain closed form solutions for the joint density of the time of ruin and the deficit at ruin. A consequence of this is that we obtain explicit formulae for finite time ruin probabilities which are of a different form to those presented by Garcia (2005) for these individual claim amount distributions.

\section{3. $\operatorname{ErLANG}(2, \beta)$ Claims}

Let us first consider the case when the individual claim amount distribution is Erlang(2) with scale parameter $\beta$, so that $f(x)=\beta^{2} x e^{-\beta x}$. Then by applying equation (2) we obtain 


$$
\left.\frac{\lambda}{c} \int_{0}^{\infty} e^{-\rho t} \int_{t}^{\infty} e^{-s(y-t)} f(y) d y d t=\frac{\lambda}{c} \mid \frac{1}{\rho+\beta}\left(\frac{\beta}{\beta+s}\right)^{2}+\frac{\beta}{(\rho+\beta)^{2}} \frac{\beta}{\beta+s}\right\rfloor,
$$

so that

$$
w(0, y, t)=h(0, t) \beta^{2} y e^{-\beta y}+k(0, t) \beta e^{-\beta y}
$$

where

$$
\tilde{h}(0, \delta) \stackrel{\text { def }}{=} \int_{0}^{\infty} e^{-\delta t} h(0, t) d t=\frac{\lambda}{c} \frac{1}{\rho+\beta}
$$

and

$$
\tilde{k}(0, \delta) \stackrel{\operatorname{def}}{=} \int_{0}^{\infty} e^{-\delta t} k(0, t) d t=\frac{\lambda}{c} \frac{\beta}{(\rho+\beta)^{2}} .
$$

Further, from equation (5) with $n=2$ and $q_{2}=1$ we have (with a change of notation)

$$
w(u, y, t)=h(u, t) \beta^{2} y e^{-\beta y}+k(u, t) \beta e^{-\beta y}
$$

where $h(u, t)$ and $k(u, t)$ are functions that we will now identify.

Inserting formula (6) in equation (1) we get

$$
\begin{aligned}
& h(u, t) \beta^{2} y e^{-\beta y}+k(u, t) \beta e^{-\beta y} \\
= & \int_{0}^{t} \int_{0}^{u} h(0, \tau) \beta^{2} x e^{-\beta x}\left(h(u-x, t-\tau) \beta^{2} y e^{-\beta y}+k(u-x, t-\tau) \beta e^{-\beta y}\right) d x d \tau \\
& +\int_{0}^{t} \int_{0}^{u} k(0, \tau) \beta e^{-\beta x}\left(h(u-x, t-\tau) \beta^{2} y e^{-\beta y}+k(u-x, t-\tau) \beta e^{-\beta y}\right) d x d \tau \\
& +h(0, t) \beta^{2}(y+u) e^{-\beta(y+u)}+k(0, t) \beta e^{-\beta(u+y)},
\end{aligned}
$$

giving

$$
h(u, t)=\int_{0}^{t} \int_{0}^{u}\left(h(0, \tau) \beta^{2} x e^{-\beta x}+k(0, \tau) \beta e^{-\beta x}\right) h(u-x, t-\tau) d x d \tau+h(0, t) e^{-\beta u}
$$

and

$$
\begin{aligned}
k(u, t)= & \int_{0}^{t} \int_{0}^{u}\left(h(0, \tau) \beta^{2} x e^{-\beta x}+k(0, \tau) \beta e^{-\beta x}\right) k(u-x, t-\tau) d x d \tau \\
& +h(0, t) \beta u e^{-\beta u}+k(0, t) e^{-\beta u} .
\end{aligned}
$$


Then defining

$$
\tilde{h}(s, \delta)=\int_{0}^{\infty} \int_{0}^{\infty} e^{-s u-\delta t} h(u, t) d t d u
$$

with a similar definition for the bivariate Laplace transform of $k(u, t)$, we obtain

$$
\tilde{h}(s, \delta)=\left(\tilde{h}(0, \delta)\left(\frac{\beta}{\beta+s}\right)^{2}+\tilde{k}(0, \delta) \frac{\beta}{\beta+s}\right) \tilde{h}(s, \delta)+\tilde{h}(0, \delta) \frac{1}{\beta+s}
$$

giving

$$
\begin{aligned}
\tilde{h}(s, \delta) & =\frac{\tilde{h}(0, \delta) \frac{1}{\beta+s}}{1-\tilde{h}(0, \delta)\left(\frac{\beta}{\beta+s}\right)^{2}-\tilde{k}(0, \delta) \frac{\beta}{\beta+s}} \\
& =\tilde{h}(0, \delta) \frac{1}{\beta+s} \sum_{n=0}^{\infty}\left(\tilde{h}(0, \delta)\left(\frac{\beta}{\beta+s}\right)^{2}+\tilde{k}(0, \delta) \frac{\beta}{\beta+s}\right)^{n} \\
& =\frac{1}{\beta} \sum_{n=0}^{\infty} \sum_{r=0}^{n}\left(\begin{array}{l}
n \\
r
\end{array}\right) \tilde{h}(0, \delta)^{r+1} \tilde{k}(0, \delta)^{n-r}\left(\frac{\beta}{\beta+s}\right)^{n+r+1} .
\end{aligned}
$$

Considering formula (8), the term in $s$ inverts to

$$
\frac{\beta^{n+r+1} u^{n+r} e^{-\beta u}}{(n+r) !}
$$

while

$$
\tilde{h}(0, \delta)^{r+1} \tilde{k}(0, \delta)^{n-r}=\left(\frac{\lambda}{c}\right)^{n+1} \frac{\beta^{n-r}}{(\rho+\beta)^{2 n-r+1}}
$$

is the Laplace transform with transform parameter $\rho$ of

$$
\left(\frac{\lambda}{c}\right)^{n+1} \frac{\beta^{n-r} t^{2 n-r} e^{-\beta t}}{(2 n-r) !}
$$

and hence by formula (3) is the Laplace transform with transform parameter $\delta$ of

$$
\left(\frac{\lambda}{c}\right)^{n+1} \beta^{n-r}\left(c e^{-\lambda t} \frac{(c t)^{2 n-r} e^{-\beta c t}}{(2 n-r) !}+\int_{0}^{c t} \frac{y}{t} g(c t-y, t) \frac{y^{2 n-r} e^{-\beta y}}{(2 n-r) !} d y\right) .
$$


Consider first the integral term in formula (9). Inserting for $g$, it becomes

$$
\begin{aligned}
& \sum_{m=1}^{\infty} e^{-\lambda t} \frac{\lambda^{m} t^{m-1}}{m !} \int_{0}^{c t} \frac{\beta^{2 m}(c t-y)^{2 m-1} e^{-\beta(c t-y)}}{(2 m-1) !} \frac{y^{2 n-r+1} e^{-\beta y}}{(2 n-r) !} d y \\
= & e^{-(\lambda+\beta c) t} \sum_{m=1}^{\infty} \frac{\left(\lambda \beta^{2}\right)^{m} t^{m-1}}{m !} \int_{0}^{c t} \frac{(c t-y)^{2 m-1} y^{2 n-r+1}}{(2 m-1) !(2 n-r) !} d y \\
= & e^{-(\lambda+\beta c) t}(c t)^{2 n-r} \sum_{m=1}^{\infty} \frac{\left(\lambda \beta^{2}\right)^{m} t^{m-1}(c t)^{2 m+1}}{m !} \frac{2 n-r+1}{(2 m+2 n-r+1) !}
\end{aligned}
$$

and the summand at $m=0$ is $c /(2 n-r)$ !, so that we can combine the terms in formula (9) to get

$$
\left(\frac{\lambda}{c}\right)^{n+1} \beta^{n-r} e^{-(\lambda+\beta c) t}(c t)^{2 n-r} \sum_{m=0}^{\infty} \frac{\left(\lambda \beta^{2}\right)^{m} t^{m-1}(c t)^{2 m+1}}{m !} \frac{2 n-r+1}{(2 m+2 n-r+1) !} .
$$

For ease of computation, we now write our solution in terms of special functions. We first note that for integer $j$,

$$
(2 m+j) !=4^{m} j !\left(\frac{j+2}{2}\right)_{m}\left(\frac{j+1}{2}\right)_{m}
$$

where $(a)_{n}=\Gamma(a+n) / \Gamma(a)$ is Pochhammer's symbol, so that we can rewrite formula (10) as

$$
\begin{aligned}
& c\left(\frac{\lambda}{c}\right)^{n+1} \beta^{n-r} e^{-(\lambda+\beta c) t}(c t)^{2 n-r} \sum_{m=0}^{\infty} \frac{\left(\lambda \beta^{2} c^{2} t^{3}\right)}{m !} \frac{2 n-r+1}{4^{m}(2 n-r+1) !\left(\frac{2 n-r+3}{2}\right)_{m}\left(\frac{2 n-r+2}{2}\right)_{m}} \\
= & c\left(\frac{\lambda}{c}\right)^{n+1} \frac{\beta^{n-r} e^{-(\lambda+\beta c) t}(c t)^{2 n-r}}{(2 n-r) !}{ }_{0} F_{2}\left(\frac{2 n-r+2}{2}, \frac{2 n-r+3}{2} ; \frac{\lambda \beta^{2} c^{2} t^{3}}{4}\right)
\end{aligned}
$$

where

$$
{ }_{p} F_{q}\left(B_{1}, B_{2}, \ldots B_{p}, C_{1}, C_{2}, \ldots C_{q} ; Z\right)=\sum_{m=0}^{\infty} \frac{\left(B_{1}\right)_{m}\left(B_{2}\right)_{m} \ldots\left(B_{p}\right)_{m}}{\left(C_{1}\right)_{m}\left(C_{2}\right)_{m} \ldots\left(C_{q}\right)_{m}} \frac{Z^{m}}{m !}
$$

is the generalised hypergeometric function. 
Finally,

$$
\begin{aligned}
h(u, t)=\frac{c}{\beta} \sum_{n=0}^{\infty} & \sum_{r=0}^{n}\left(\begin{array}{l}
n \\
r
\end{array}\right) \frac{\beta^{n+r+1} u^{n+r} e^{-\beta u}}{(n+r) !}\left(\frac{\lambda}{c}\right)^{n+1} \frac{\beta^{n-r} e^{-(\lambda+\beta c) t}(c t)^{2 n-r}}{(2 n-r) !} \\
& \times{ }_{0} F_{2}\left(\frac{2 n-r+2}{2}, \frac{2 n-r+3}{2} ; \frac{\lambda \beta^{2} c^{2} t^{3}}{4}\right) \\
= & \lambda e^{-\beta u-(\lambda+\beta c) t} \sum_{n=0}^{\infty}\left(\frac{\lambda}{c}\right)^{n}\left(\beta^{2} u\right)^{n} \sum_{r=0}^{n}\left(\begin{array}{l}
n \\
r
\end{array}\right) \frac{u^{r}}{(n+r) !} \frac{(c t)^{2 n-r}}{(2 n-r) !} \\
& \times{ }_{0} F_{2}\left(\frac{2 n-r+2}{2}, \frac{2 n-r+3}{2} ; \frac{\lambda \beta^{2} c^{2} t^{3}}{4}\right)
\end{aligned}
$$

with

$$
h(0, t)=\lambda e^{-(\lambda+c \beta) t}{ }_{0} F_{2}\left(1, \frac{3}{2} ; \frac{\lambda \beta^{2} c^{2} t^{3}}{4}\right) .
$$

The solution for $k(u, t)$ is obtained by exactly the same approach. We obtain

$$
\begin{gathered}
k(u, t)=\lambda \beta u e^{-\beta u-(\lambda+\beta c) t} \sum_{n=0}^{\infty}\left(\frac{\lambda}{c}\right)^{n}\left(\beta^{2} u\right)^{n} \sum_{r=0}^{n}\left(\begin{array}{l}
n \\
r
\end{array}\right) \frac{u^{r}}{(n+r+1) !} \frac{(c t)^{2 n-r}}{(2 n-r) !} \\
\quad \times{ }_{0} F_{2}\left(\frac{2 n-r+2}{2}, \frac{2 n-r+3}{2} ; \frac{\lambda \beta^{2} c^{2} t^{3}}{4}\right) \\
+\frac{\beta e^{-\beta u-(\lambda+\beta c) t}}{t} \sum_{n=0}^{\infty}\left(\frac{\lambda}{c}\right)^{n+1}\left(\beta^{2} u\right)^{n} \sum_{r=0}^{n}\left(\begin{array}{l}
n \\
r
\end{array}\right) \frac{u^{r}}{(n+r) !} \frac{(c t)^{2 n-r+2}}{(2 n-r+1) !} \\
\quad \times{ }_{0} F_{2}\left(\frac{2 n-r+3}{2}, \frac{2 n-r+4}{2} ; \frac{\lambda \beta^{2} c^{2} t^{3}}{4}\right),
\end{gathered}
$$

with

$$
k(0, t)=\lambda \beta c t e^{-(\lambda+c \beta) t}{ }_{0} F_{2}\left(\frac{3}{2}, 2 ; \frac{\lambda \beta^{2} c^{2} t^{3}}{4}\right) .
$$

Further, we can compute the density of the time of ruin as $w(u, t)=h(u, t)+$ $k(u, t)$.

Figures 1 to 3 illustrate the application of these formulae. In each figure, $\beta=2, \lambda=1$ and $c=1.1$. The values of $u$ are 0 in Figure 1, 10 in Figure 2 and 20 in Figure 3. All values were calculated using Mathematica, which includes the generalised hypergeometric function as a supplied function, and infinite sums were truncated at a suitably high level. Although it is clear from the above formulae that $h(u, t)$ and $k(u, t)$ can be written in terms of Erlang densities, it is straightforward to integrate these functions numerically to obtain values of 


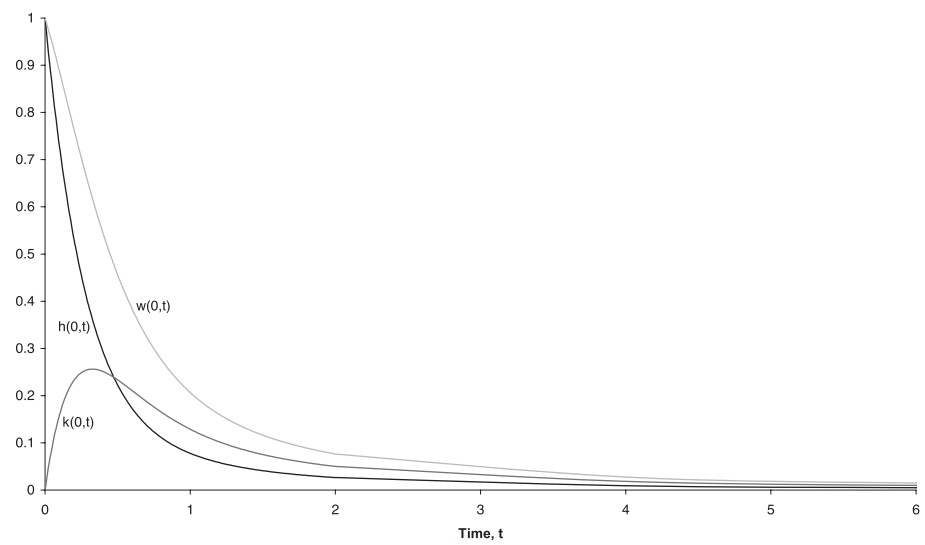

Figure 1: Erlang(2) claims, $u=0$.

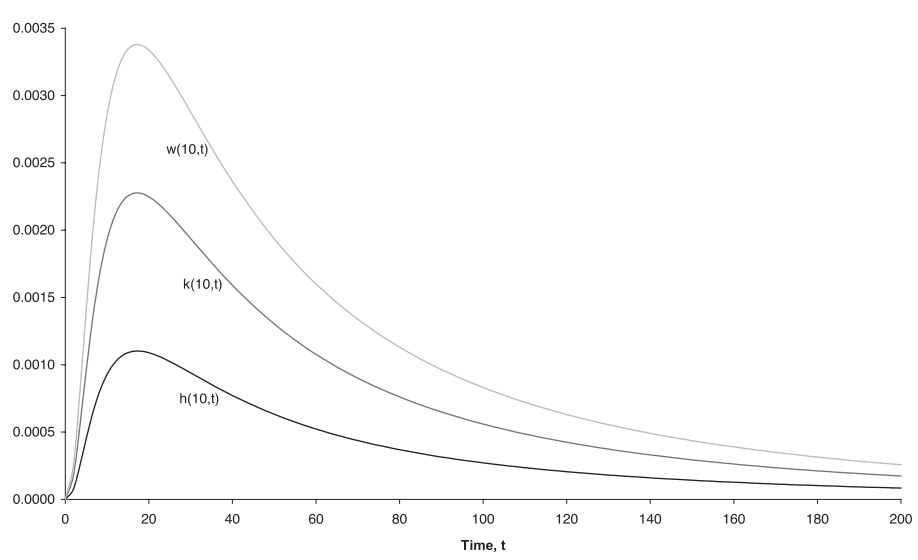

FIGURE 2: Erlang(2) claims, $u=10$.

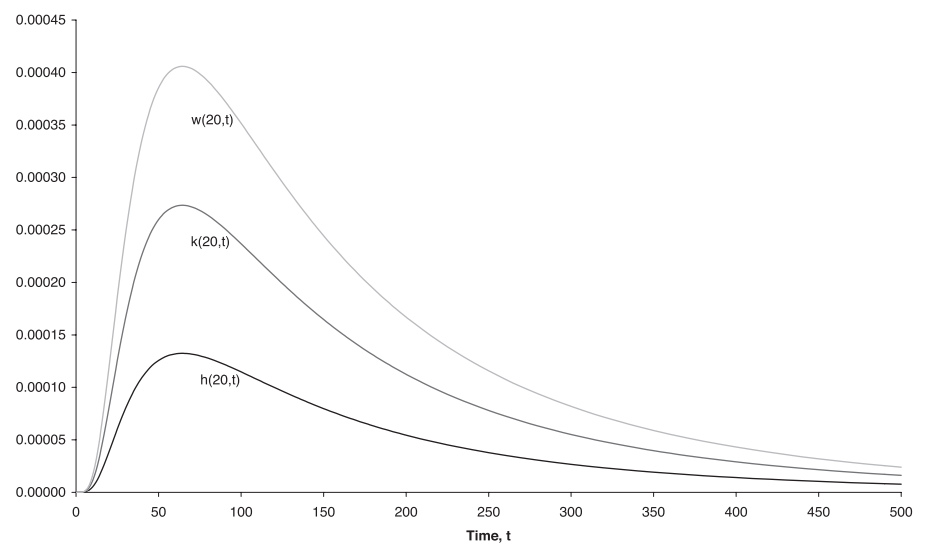

FIGURE 3: Erlang(2) claims, $u=20$. 
TABLE 1

VALUES OF $W(10, t, y)$, ERLANG(2) CLAIMS

\begin{tabular}{l|c|c|c|c}
\hline \hline & $y=1$ & $y=2$ & $y=3$ & $y \rightarrow \infty$ \\
\hline$t=10$ & 0.0107 & 0.0131 & 0.0136 & 0.0137 \\
$t=20$ & 0.0360 & 0.0444 & 0.0460 & 0.0464 \\
$t=30$ & 0.0603 & 0.0744 & 0.0771 & 0.0776 \\
$t=40$ & 0.0806 & 0.0994 & 0.1030 & 0.1038 \\
$t=50$ & 0.0972 & 0.1199 & 0.1243 & 0.1252 \\
$t=60$ & 0.1109 & 0.1368 & 0.1418 & 0.1428 \\
$t=70$ & 0.1222 & 0.1508 & 0.1563 & 0.1575 \\
$t=80$ & 0.1318 & 0.1626 & 0.1685 & 0.1698 \\
$t=90$ & 0.1399 & 0.1726 & 0.1789 & 0.1802 \\
$t=100$ & 0.1469 & 0.1812 & 0.1878 & 0.1892 \\
\hline \hline
\end{tabular}

the joint distribution function and of the finite time ruin probability. Values of $W(10, t, y)$ are shown in Table 1 for a range of values for $t$ and $y$ for the same parameters as in Figures 1 to 3.

\section{Mixed exponential Claims}

We now consider the case when individual claims have a mixed exponential distribution with density

$$
f(x)=p \alpha e^{-\alpha x}+q \beta e^{-\beta x}
$$

where $p+q=1,0<p<1$ and $\alpha<\beta$. Then from formula (4), we can write

$$
w(u, y, t)=\eta(u, t) \alpha e^{-\alpha y}+\kappa(u, t) \beta e^{-\beta y}
$$

and we will now determine $\eta(u, t)$ and $\kappa(u, t)$. From equation (2) we obtain

$$
\tilde{\eta}(0, \delta) \stackrel{\text { def }}{=} \int_{0}^{\infty} e^{-\delta t} \eta(0, t) d t=\frac{\lambda}{c} \frac{p}{\rho+\alpha}
$$

and

$$
\tilde{\kappa}(0, \delta) \stackrel{\text { def }}{=} \int_{0}^{\infty} e^{-\delta t} \kappa(0, t) d t=\frac{\lambda}{c} \frac{q}{\rho+\beta} .
$$

The method of solution is essentially the same as in the previous section, but the details are more complicated. We first insert expression (11) into equation (1), giving 


$$
\begin{aligned}
& \eta(u, t) \alpha e^{-\alpha y}+\kappa(u, t) \beta e^{-\beta y} \\
= & \int_{0}^{t} \int_{0}^{u}\left(\eta(0, \tau) \alpha e^{-\alpha x}+\kappa(0, \tau) \beta e^{-\beta x}\right) \eta(u-x, t-\tau) \alpha e^{-\alpha y} d x d \tau \\
& +\int_{0}^{t} \int_{0}^{u}\left(\eta(0, \tau) \alpha e^{-\alpha x}+\kappa(0, \tau) \beta e^{-\beta x}\right) \kappa(u-x, t-\tau) \beta e^{-\beta y} d x d \tau \\
& +\eta(0, t) \alpha e^{-\alpha(u+y)}+\kappa(0, t) \beta e^{-\beta(u+y)} .
\end{aligned}
$$

Then

$\eta(u, t)=\int_{0}^{t} \int_{0}^{u}\left(\eta(0, \tau) \alpha e^{-\alpha x}+\kappa(0, \tau) \beta e^{-\beta x}\right) \eta(u-x, t-\tau) d x d \tau+\eta(0, t) e^{-\alpha u}$.

Let

$$
\tilde{\tilde{\eta}}(s, \delta)=\int_{0}^{\infty} \int_{0}^{\infty} e^{-s u-\delta t} \eta(u, t) d t d u \text { and } \tilde{\eta}(0, \delta)=\int_{0}^{\infty} e^{-\delta t} \eta(0, t) d t,
$$

with similar notation for the Laplace transforms of $\kappa(u, t)$ and $\kappa(0, t)$. Then

$$
\tilde{\tilde{\eta}}(s, \delta)=\left(\tilde{\eta}(0, \delta) \frac{\alpha}{\alpha+s}+\tilde{\kappa}(0, \delta) \frac{\beta}{\beta+s}\right) \tilde{\tilde{\eta}}(s, \delta)+\tilde{\eta}(0, \delta) \frac{1}{\alpha+s}
$$

giving

$$
\begin{aligned}
\tilde{\tilde{\eta}}(s, \delta) & =\frac{\tilde{\eta}(0, \delta) \frac{1}{\alpha+s}}{1-\tilde{\eta}(0, \delta) \frac{\alpha}{\alpha+s}-\tilde{\kappa}(0, \delta) \frac{\beta}{\beta+s}} \\
& =\tilde{\eta}(0, \delta) \frac{1}{\alpha+s} \sum_{n=0}^{\infty}\left(\tilde{\eta}(0, \delta) \frac{\alpha}{\alpha+s}+\tilde{\kappa}(0, \delta) \frac{\beta}{\beta+s}\right)^{n} \\
& =\frac{1}{\alpha} \sum_{n=0}^{\infty} \sum_{k=0}^{n}\left(\begin{array}{l}
n \\
k
\end{array}\right)\left(\tilde{\eta}(0, \delta) \frac{\alpha}{\alpha+s}\right)^{k+1}\left(\tilde{\kappa}(0, \delta) \frac{\beta}{\beta+s}\right)^{n-k}
\end{aligned}
$$

Inversion of

$$
\left(\frac{\alpha}{\alpha+s}\right)^{k+1}\left(\frac{\beta}{\beta+s}\right)^{n-k}
$$

gives

$$
\begin{aligned}
& \int_{0}^{u} \frac{\alpha^{k+1} x^{k} e^{-\alpha x}}{\Gamma(k+1)} \frac{\beta^{n-k}(u-x)^{n-k-1} e^{-\beta(u-x)}}{\Gamma(n-k)} d x \\
= & \frac{\alpha^{k+1} \beta^{n-k} e^{-\beta u}}{\Gamma(k+1) \Gamma(n-k)} \int_{0}^{u} x^{k} e^{-(\alpha-\beta) x}(u-x)^{n-k-1} d x
\end{aligned}
$$




$$
\begin{aligned}
& =\frac{\alpha^{k+1} \beta^{n-k} e^{-\beta u}}{\Gamma(k+1) \Gamma(n-k)} \int_{0}^{1}(u t)^{k} e^{-(\alpha-\beta) u t}(u-u t)^{n-k-1} u d t \\
& =e^{-\beta u}\left(\frac{\alpha}{\beta}\right)^{k} \frac{\alpha(\beta u)^{n}}{\Gamma(n+1)}{ }_{1} F_{1}(k+1, n+1,(\beta-\alpha) u) .
\end{aligned}
$$

(See Abramowitz and Stegun (1965, p. 505).)

Now consider inversion of $(\tilde{\eta}(0, \delta))^{k+1}(\tilde{\kappa}(0, \delta))^{n-k}$. We have

$$
\begin{aligned}
& (\tilde{\eta}(0, \delta))^{k+1}(\tilde{\kappa}(0, \delta))^{n-k} \\
= & \left(\frac{\lambda}{c}\right)^{n+1}\left(\frac{p}{\rho+\alpha}\right)^{k+1}\left(\frac{q}{\rho+\beta}\right)^{n-k} \\
= & \left(\frac{\lambda}{c}\right)^{n+1} p^{k+1} q^{n-k} \int_{0}^{\infty} e^{-\rho t} \int_{0}^{t} \frac{x^{k} e^{-\alpha x}}{\Gamma(k+1)} \frac{(t-x)^{n-k-1} e^{-\beta(t-x)}}{\Gamma(n-k)} d x d t \\
= & \left(\frac{\lambda}{c}\right)^{n+1} p^{k+1} q^{n-k} \int_{0}^{\infty} e^{-\rho t} \frac{t^{n} e^{-\beta t}}{\Gamma(n+1)}{ }_{1} F_{1}(k+1, n+1,(\beta-\alpha) t) d t .
\end{aligned}
$$

By formula (3) this is the Laplace transform with transform parameter $\delta$ of

$$
\begin{aligned}
& \left(\frac{\lambda}{c}\right)^{n+1} p^{k+1} q^{n-k}\left(c e^{-\lambda t} \frac{(c t)^{n} e^{-\beta c t}}{\Gamma(n+1)}{ }_{1} F_{1}(k+1, n+1,(\beta-\alpha) c t)\right. \\
& \left.+\int_{0}^{c t} \frac{y}{t} g(c t-y, t) \frac{y^{n} e^{-\beta y}}{\Gamma(n+1)}{ }_{1} F_{1}(k+1, n+1,(\beta-\alpha) y) d y\right) .
\end{aligned}
$$

To evaluate the above integral we must insert for $g$ and hence we need an expression for $f^{n^{*}}$. Following Willmot and Woo (2007) we can write

$$
f^{n^{*}}(x)=\sum_{j=0}^{\infty} \gamma_{n, j} e(n+j, \beta ; x)
$$

where $e(n, \beta ; x)$ denotes the $\operatorname{Erlang}(n)$ density with scale parameter $\beta$ and

$$
\gamma_{n, j}=q^{n}(1-\alpha / \beta)^{j} \sum_{r=0}^{n}\left(\begin{array}{l}
n \\
r
\end{array}\right) \frac{(r)_{j}}{j !}\left(\frac{\alpha p}{\beta q}\right)^{r} .
$$

Further, let us write

$$
{ }_{1} F_{1}(k+1, n+1,(\beta-\alpha) y)=\sum_{i=0}^{\infty} d_{n, k, i} y^{i}
$$


where

$$
d_{n, k, i}=\frac{(k+1)_{i}}{(n+1)_{i}} \frac{(\beta-\alpha)^{i}}{i !} .
$$

Then

$$
\begin{aligned}
& \int_{0}^{c t} \frac{y}{t} g(c t-y, t) \frac{y^{n} e^{-\beta y}}{\Gamma(n+1)}{ }_{1} F_{1}(k+1, n+1,(\beta-\alpha) y) d y \\
= & \sum_{m=1}^{\infty} e^{-\lambda t} \frac{(\lambda t)^{m}}{m !} \int_{0}^{c t} \sum_{j=0}^{\infty} \gamma_{m, j} e(m+j, \beta ; c t-y) \frac{y}{t} \frac{y^{n} e^{-\beta y}}{n !} \sum_{i=0}^{\infty} d_{n, k, i} y^{i} d y \\
= & \sum_{m=1}^{\infty} e^{-(\lambda+\beta c) t} \frac{\lambda^{m} t^{m-1}}{m !} \sum_{j=0}^{\infty} \gamma_{m, j} \sum_{i=0}^{\infty} d_{n, k, i} \int_{0}^{c t} \frac{\beta^{m+j}(c t-y)^{m+j-1}}{\Gamma(m+j)} \frac{y^{n+i+1}}{n !} d y \\
= & \frac{1}{n !} \sum_{m=1}^{\infty} e^{-(\lambda+\beta c) t} \frac{\lambda^{m} t^{m-1}}{m !} \sum_{j=0}^{\infty} \gamma_{m, j} \sum_{i=0}^{\infty} d_{n, k, i} \frac{\beta^{m+j}}{\Gamma(m+j)} \int_{0}^{c t}(c t-y)^{m+j-1} y^{n+i+1} d y \\
= & \frac{1}{n !} \sum_{m=1}^{\infty} e^{-(\lambda+\beta c) t} \frac{\lambda^{m} t^{m-1}}{m !} \sum_{j=0}^{\infty} \gamma_{m, j} \sum_{i=0}^{\infty} d_{n, k, i} \frac{\beta^{m+j}}{\Gamma(m+j)} \\
& \times(c t)^{m+j+n+i+1} \frac{\Gamma(m+j) \Gamma(n+i+2)}{\Gamma(m+j+n+i+2)} \\
= & \frac{c^{n+1}}{n !} \sum_{m=1}^{\infty} \frac{\lambda^{m}}{m !} \sum_{j=0}^{\infty} \gamma_{m, j}(\beta c)^{m+j} \sum_{i=0}^{\infty} \frac{d_{n, k, i} c^{i}}{(n+i+2)_{m+j}} e^{-(\lambda+c \beta) t} t^{2 m+j+n+i} \\
= & \frac{c^{n+1}}{n !} \sum_{m=1}^{\infty} \frac{\lambda^{m}}{m !} \sum_{j=0}^{\infty} \gamma_{m, j}(\beta c)^{m+j} \sum_{i=0}^{\infty} \frac{d_{n, k, i} c^{i}}{(n+i+2)_{m+j}} \\
& \times \frac{\Gamma(2 m+j+n+i+1)}{(\lambda+c \beta)^{2 m+j+n+i+1} e(2 m+j+n+i+1, \lambda+c \beta ; t)} \\
= & \left(\frac{c}{\lambda+c \beta}\right)^{n+1} \sum_{m=1}^{\infty} \frac{1}{m !}\left(\frac{\lambda}{\lambda+c \beta}\right)^{m} \sum_{j=0}^{\infty} \gamma_{m, j}\left(\frac{c \beta}{\lambda+c \beta}\right)^{m+j} \\
& \times \sum_{i=0}^{\infty} \frac{d_{n, k, i}}{(n+i+2)_{m+j}}\left(\frac{c}{\lambda+c \beta}\right)^{i} \frac{\Gamma(2 m+j+n+i+1)}{n !} e(2 m+j+n+i+1, \lambda+c \beta ; t) \\
& N o w
\end{aligned}
$$

Now note that

$$
\begin{aligned}
& \frac{\Gamma(2 m+j+n+i+1)}{n !} \\
= & \frac{\Gamma(2 m+j+n+i+1)}{\Gamma(2 m+j+n+1)} \frac{\Gamma(2 m+j+n+1)}{\Gamma(2 m+n+1)} \frac{\Gamma(2 m+n+1)}{\Gamma(n+1)} \\
= & (2 m+j+n+1)_{i}(2 m+n+1)_{j}(n+1)_{2 m}
\end{aligned}
$$


so that for computational purposes,

$$
\begin{aligned}
& \int_{0}^{c t} \frac{y}{t} g(c t-y, t) \frac{y^{n} e^{-\beta y}}{\Gamma(n+1)}{ }_{1} F_{1}(k+1, n+1,(\beta-\alpha) y) d y \\
= & \left(\frac{c}{\lambda+c \beta}\right)^{n+1} \sum_{m=1}^{\infty} \frac{(n+1)_{2 m}}{m !}\left(\frac{\lambda}{\lambda+c \beta}\right)^{m} \sum_{j=0}^{\infty} \gamma_{m, j}\left(\frac{c \beta}{\lambda+c \beta}\right)^{m+j}(2 m+n+1)_{j} \\
& \times \sum_{i=0}^{\infty} \frac{d_{n, k, i}(2 m+j+n+1)_{i}}{(n+i+2)_{m+j}}\left(\frac{c}{\lambda+c \beta}\right)^{i} e(2 m+j+n+i+1, \lambda+c \beta ; t) .
\end{aligned}
$$

Further,

$$
\begin{aligned}
& \int_{0}^{t} c e^{-\lambda \tau} \frac{(c \tau)^{n} e^{-\beta c \tau}}{\Gamma(n+1)}{ }_{1} F_{1}(k+1, n+1,(\beta-\alpha) c \tau) d \tau \\
= & \frac{c^{n+1}}{n !(\lambda+c \beta)^{n+1}} \sum_{j=0}^{\infty} \frac{(k+1)_{j}}{(n+1)_{j}} \frac{[(\beta-\alpha) c]^{j}}{j !(\lambda+c \beta)^{j}} \Gamma(n+j+1) E(n+j+1,(\lambda+c \beta) t)
\end{aligned}
$$

and

$$
\begin{aligned}
& \int_{0}^{t} \int_{0}^{c \tau} \frac{y}{\tau} g(c \tau-y, \tau) \frac{y^{n} e^{-\beta y}}{\Gamma(n+1)}{ }_{1} F_{1}(k+1, n+1,(\beta-\alpha) y) d y d \tau \\
= & \left(\frac{c}{\lambda+c \beta}\right)^{n+1} \sum_{m=1}^{\infty} \frac{(n+1)_{2 m}}{m !}\left(\frac{\lambda}{\lambda+c \beta}\right)^{m} \sum_{j=0}^{\infty} \gamma_{m, j}\left(\frac{c \beta}{\lambda+c \beta}\right)^{m+j}(2 m+n+1)_{j} \\
& \times \sum_{i=0}^{\infty} \frac{d_{n, k, i}(2 m+j+n+1)_{i}}{(n+i+2)_{m+j}}\left(\frac{c}{\lambda+c \beta}\right)^{i} E(2 m+j+n+i+1, \lambda+c \beta ; t),
\end{aligned}
$$

where $E(n, \beta ; x)$ denotes the $\operatorname{Erlang}(n)$ distribution function with scale parameter $\beta$.

Now let the sum of expressions (13) and (14) be denoted by $\zeta_{n, k}(t)$. Then

$$
\begin{aligned}
H(u, t) & \stackrel{\text { def }}{=} \int_{0}^{t} \eta(u, \tau) d \tau \\
& =\frac{\lambda p e^{-\beta u}}{c} \sum_{n=0}^{\infty} \frac{(\lambda \beta q u / c)^{n}}{n !} \sum_{k=0}^{n}\left(\begin{array}{l}
n \\
k
\end{array}\right)\left(\frac{\alpha p}{\beta q}\right)^{k} F_{1}(k+1, n+1,(\beta-\alpha) u) \zeta_{n, k}(t)
\end{aligned}
$$

We can take the same approach to obtain $\kappa(u, t)$, but we omit the details. We obtain 


$$
\begin{aligned}
K(u, t) & \stackrel{\text { def }}{=} \int_{0}^{t} \kappa(u, \tau) d \tau \\
& =\frac{\lambda q e^{-\beta u}}{c} \sum_{n=0}^{\infty} \frac{(\lambda p \alpha u / c)^{n}}{n !} \sum_{k=0}^{n}\left(\begin{array}{l}
n \\
k
\end{array}\right)\left(\frac{\beta q}{\alpha p}\right)^{k}{ }_{1} F_{1}(n-k, n+1,(\beta-\alpha) u) \zeta_{n, k}^{*}(t),
\end{aligned}
$$

where

$$
\begin{aligned}
& \zeta_{n, k}^{*}(t)=\frac{c^{n+1}}{n !(\lambda+c \beta)^{n+1}} \sum_{j=0}^{\infty} \frac{(n-k)_{j}}{(n+1)_{j}} \frac{[(\beta-\alpha) c]^{j}}{j !(\lambda+c \beta)^{j}} \Gamma(n+j+1) E(n+j+1,(\lambda+c \beta) t) \\
& +\left(\frac{c}{\lambda+c \beta}\right)^{n+1} \sum_{m=1}^{\infty} \frac{(n+1)_{2 m}}{m !}\left(\frac{\lambda}{\lambda+c \beta}\right)^{m} \sum_{j=0}^{\infty} \gamma_{m, j}\left(\frac{c \beta}{\lambda+c \beta}\right)^{m+j}(2 m+n+1)_{j} \\
& \times \sum_{i=0}^{\infty} \frac{d_{n, k, i}^{*}(2 m+j+n+1)_{i}}{(n+i+2)_{m+j}}\left(\frac{c}{\lambda+c \beta}\right)^{i} E(2 m+j+n+i+1, \lambda+c \beta ; t),
\end{aligned}
$$

with $d_{n, k, i}^{*}$ defined as

$$
d_{n, k, i}^{*}=\frac{(n-k)_{i}}{(n+1)_{i}} \frac{(\beta-\alpha)^{i}}{i !} .
$$

Further, $\psi(u, t)=H(u, t)+K(u, t)$.

The above formulae for $H(u, t)$ and $K(u, t)$ involve infinite sums whose summands comprise simple functions (and the same is true for $\eta(u, t)$ and $\kappa(u, t)$ ). In applying these formulae, we generally found it easier to compute $H(u, t)$ and $K(u, t)$ (although this may simply reflect the author's programming skills), and results based on these are shown in Tables 2 to 4 . For small values of $u$ and $t$, evaluation of $H(u, t)$ and $K(u, t)$ posed no problems and computer run times were short. For the larger values of $u$ and $t$ in Tables 2 to 4, computer run times are very lengthy (several hours) and care has to be exercised in computer programs to avoid numerical underflow or overflow. To illustrate our formulae, we

TABLE 2

VALUES WHEN $u=0$, MiXed EXPONENTIAL CLAIMS

\begin{tabular}{l|c|c|c|c|c|c}
\hline \hline & $H(0, t)$ & $K(0, t)$ & $\psi(0, t)$ & $W(0,1, t)$ & $W(0,3, t)$ & $W(0,5, t)$ \\
\hline$t=10$ & 0.4640 & 0.2863 & 0.7503 & 0.4301 & 0.6460 & 0.7122 \\
$t=20$ & 0.5142 & 0.2924 & 0.8066 & 0.4551 & 0.6911 & 0.7643 \\
$t=30$ & 0.5365 & 0.2950 & 0.8316 & 0.4662 & 0.7111 & 0.7875 \\
$t=40$ & 0.6497 & 0.2966 & 0.8463 & 0.4727 & 0.7229 & 0.8012 \\
$t=50$ & 0.5587 & 0.2976 & 0.8563 & 0.4771 & 0.7309 & 0.8104 \\
\hline \hline
\end{tabular}


TABLE 3

VALUeS WHen $u=10$, MiXed EXPONENTIAL ClAimS

\begin{tabular}{c|c|c|c|c|c|c}
\hline \hline & $H(10, t)$ & $K(10, t)$ & $\psi(10, t)$ & $W(10,1, t)$ & $W(10,3, t)$ & $W(10,5, t)$ \\
\hline$t=10$ & 0.0644 & 0.0068 & 0.0712 & 0.0312 & 0.0568 & 0.0659 \\
$t=20$ & 0.1281 & 0.0142 & 0.1422 & 0.0626 & 0.1136 & 0.1317 \\
$t=30$ & 0.1754 & 0.0196 & 0.1950 & 0.0860 & 0.1558 & 0.1806 \\
$t=40$ & 0.2110 & 0.0237 & 0.2347 & 0.1035 & 0.1876 & 0.2174 \\
$t=50$ & 0.2387 & 0.0269 & 0.2656 & 0.1172 & 0.2123 & 0.2460 \\
\hline \hline
\end{tabular}

TABLE 4

VALUeS When $u=20$, MiXed EXPONENTIAL Claims

\begin{tabular}{c|c|c|c|c|c|c}
\hline \hline & $H(20, t)$ & $K(20, t)$ & $\psi(20, t)$ & $W(20,1, t)$ & $W(20,3, t)$ & $W(20,5, t)$ \\
\hline$t=10$ & 0.0041 & 0.0004 & 0.0045 & 0.0020 & 0.0036 & 0.0042 \\
$t=20$ & 0.0156 & 0.0017 & 0.0173 & 0.0076 & 0.0138 & 0.0160 \\
$t=30$ & 0.0303 & 0.0033 & 0.0336 & 0.0148 & 0.0268 & 0.0311 \\
$t=40$ & 0.0453 & 0.0050 & 0.0504 & 0.0222 & 0.0402 & 0.0466 \\
$t=50$ & 0.0596 & 0.0067 & 0.0663 & 0.0292 & 0.0530 & 0.0614 \\
\hline \hline
\end{tabular}

TABLE 5

EXACT AND APPROXIMATE VALUES WHEN $u=20$, MiXed EXPONENTIAL Claims

\begin{tabular}{c|c|c|c|c}
\hline \hline & $W(20,1, t)$ & $W(20,3, t)$ & $W(20,5, t)$ & $\psi(20, t)$ \\
\hline \multirow{2}{*}{$t=10$} & 0.0020 & 0.0036 & 0.0042 & 0.0045 \\
& $(0.0020)$ & $(0.0036)$ & $(0.0042)$ & $(0.0045)$ \\
$t=30$ & 0.0148 & 0.0268 & 0.0311 & 0.0336 \\
& $(0.0148)$ & $(0.0268)$ & $(0.0311)$ & $(0.0336)$ \\
$t=50$ & 0.0292 & 0.0530 & 0.0614 & 0.0663 \\
& $(0.0292)$ & $(0.0529)$ & $(0.0614)$ & $(0.0663)$ \\
\hline \hline
\end{tabular}

have chosen the same mixed exponential distribution and parameters as Garcia (2005), namely $p=1-q=\frac{1}{3}, \alpha=\frac{1}{3}$ and $\beta=2$, with $\lambda=1$ and $c=1.1$.

As it took several hours of computing time to produce the values in Table 4, we also calculated approximations to values of $W(20, y, t)$ using the algorithm of Dickson and Waters (1992). The approximations are shown in parenthesis in Table 5, and are based on a scaling factor of 100 in the algorithm. (A higher value of the scaling factor can be used to produce better approximations.) By contrast with the computer run time required to produce exact values, the approximations were computed in minutes, and are excellent. 


\section{CONCLUDiNG REMARKS}

The approach presented in this paper easily applies in the case of exponential individual claims. It produces the same solution for $w(u, t)$ as Drekic and Willmot (2003) give, although the transform inversion problem is quite different. Similarly, if

$$
f(x)=p \beta e^{-\beta x}+(1-p) \beta^{2} x e^{-\beta x}
$$

where $0<p<1$, an explicit solution can be obtained for $w(u, y, t)$. The approach, however, becomes unwieldy for other individual claim amount distributions, for example Erlang(3), although it is possible to obtain further explicit solutions when $u=0$. Nevertheless, this approach has allowed us to obtain formulae from which the joint distribution of the time of ruin and the deficit at ruin can be calculated. It should also apply to certain Sparre Andersen models with the individual claim amount distributions considered in this paper, although a first stage in developing this idea will be to find a transform relationship equivalent to (3) for these models.

We had mixed experiences in our computations. For Erlang(2) claims, programming of formulae is straightforward and computations can be done quickly. By contrast, for our example with mixed exponential claims, programming was more complicated and computer run times were lengthy. Our comparison of exact and approximate values of $W(u, y, t)$ in Table 3 suggests that while it is appealing to have an explicit solution to a problem, in practice it may be more appropriate to compute an approximate solution.

\section{REFERENCES}

Abramowitz, M. and Stegun, I.A. (1965) Handbook of Mathematical Functions. Dover, New York.

Cheung, E.C.K., Dickson, D.C.M. and Drekic, S. (2006) Optimal dividend threshold levels for two classes of claim size distributions. Unpublished manuscript.

Dickson, D.C.M. and DREKIC, S. (2006) Optimal dividends under a ruin probability constraint. Annals of Actuarial Science, 1, 291-306.

DiCKSON, D.C.M. and WATERS, H.R. (1992) The probability and severity of ruin in finite and in infinite time. ASTIN Bulletin, 22, 177-190.

Dickson, D.C.M. and Willmot, G.E. (2005) The density of the time to ruin in the classical Poisson risk model. ASTIN Bulletin, 35, 45-60.

Dickson, D.C.M., Hughes, B.D. and Lianzeng, Z. (2005) The density of the time to ruin for a Sparre Andersen process with Erlang arrivals and exponential claims. Scandinavian Actuarial Journal, 2005(5), 358-376

DreKic, S. and Willmot, G.E. (2003) On the density and moments of the time to ruin with exponential claims. ASTIN Bulletin, 33, 11-21.

GARCIA, J.M.A. (2005) Explicit solutions for survival probabilities in the classical risk model. ASTIN Bulletin, 35, 113-130.

Gerber, H.U. (1979) An Introduction to Mathematical Risk Theory. S.S. Huebner Foundation, Philadelphia, PA. 
Gerber, H.U. and Shiu, E.S.W. (1998) On the time value of ruin. North American Actuarial Journal, 2(1), 48-78.

WiLlmot, G.E. and Woo, J.K. (2007) On the class of Erlang mixtures with risk theoretic applications. North American Actuarial Journal, 11(2), 99-115.

DAVID C.M. Dickson

Centre for Actuarial Studies

Department of Economics

University of Melbourne

Victoria 3010

Australia

E-Mail:dcmd@unimelb.edu.au 